\title{
Segurança do Paciente: uma abordagem específica no Curso Técnico em Enfermagem
}

RESUMO I Objetivou-se criar cartilha educativa para contribuir na prática de introdução da segurança do paciente e, posteriormente, fornecê-las aos hospitais e instituições de saúde e elaborar e aplicar curso extracurricular sobre segurança do paciente em curso técnico em enfermagem para contribuição na formação de um profissional qualificado. O estudo foi desenvolvido na Escola Técnica: ETEC - Joaquim Ferreira do Amaral- Jaú - Centro Paula Souza, no curso Técnico de Enfermagem envolvendo 46 alunos do $3^{\circ}$ e $4^{\circ}$ módulo a partir do segundo semestre de 2017, sendo que 36 realizaram o curso e 10 realizaram somente as avaliações pré e pós-curso. A avaliação do conhecimento e habilidades adquiridas sobre segurança do paciente no currículo tradicional do curso técnico e após curso específico sobre o tema foi realizada através de provas teóricas aplicadas antes e após o curso e de avaliação prática no final do curso (OSCE). Foi utilizado o teste t de student para verificar diferenças entre as medias das notas obtidas nos grupos que realizaram e não realizaram o curso sendo que houve diferença estatisticamente significante entre eles. Conclui-se, portanto, que uma abordagem especifica sobre o tema traz contribuições positivas para o desenvolvimento das competências previstas no plano de curso do técnico em enfermagem.

Palavras-chaves: Segurança do Paciente; Ensino; Técnico em Enfermagem.

\begin{abstract}
The aim was to create an educational booklet to contribute to the practice of introducing patient safety and subsequently provide them to hospitals and health institutions and to develop and apply an extracurricular course on patient safety in a technical nursing course to contribute to the training of a qualified professional. . The study was developed at the Technical School: ETEC - Joaquim Ferreira do Amaral- Jaú - Centro Paula Souza, in the Nursing Technician course involving 46 students from the 3rd and 4th modules from the second semester of 2017, with 36 taking the course and 10 performed only the pre- and post-course evaluations. The assessment of knowledge and skills acquired about patient safety in the traditional curriculum of the technical course and after a specific course on the topic was carried out through theoretical tests applied before and after the course and practical evaluation at the end of the course (OSCE). Student's t-test was used to verify differences between the mean scores obtained in the groups that took and did not take the course, with a statistically significant difference between them. It is concluded, therefore, that a specific approach on the subject brings positive contributions to the development of the competencies provided for in the nursing technician's course plan.
\end{abstract}

Keywords: Patient Safety; Teaching; Nursing Technician.

RESUMEN | El objetivo era crear un folleto educativo para contribuir a la práctica de introducir la seguridad del paciente y posteriormente proporcionarlos a hospitales e instituciones de salud y desarrollar y aplicar un curso extracurricular sobre seguridad del paciente en un curso de enfermería técnica para contribuir a la capacitación de un profesional calificado. El estudio se desarrolló en la Escuela Técnica: ETEC - Joaquim Ferreira do Amaral- Jaú - Centro Paula Souza, en el curso de Técnico de Enfermería que involucró a 46 estudiantes de los módulos tercero y cuarto del segundo semestre de 2017 , con 36 tomando el curso y 10 realizaron solo las evaluaciones previas y posteriores al curso. La evaluación de los conocimientos y habilidades adquiridas sobre la seguridad del paciente en el plan de estudios tradicional del curso técnico y después de un curso específico sobre el tema se llevó a cabo mediante pruebas teóricas aplicadas antes y después del curso y una evaluación práctica al final del curso (OSCE). La prueba t de Student se utilizó para verificar las diferencias entre los puntajes promedio obtenidos en los grupos que tomaron y no tomaron el curso, con una diferencia estadísticamente significativa entre ellos. Se concluye, por lo tanto, que un enfoque específico sobre el tema aporta contribuciones positivas al desarrollo de las competencias previstas en el plan de cursos del técnico de enfermería.

Palavras claves: Seguridad del Paciente; Enseñando; Tecnico de Enfermería.

\section{Giovana Cristina Serra D'Amico}

Enfermeira. Mestre em Pesquisa Clínica pela Faculdade de Medicina de Botucatu. SP/Brasil. https://orcid.org/0000-0002-3397-0850

\section{Adriana Polachini do Valle}

Médica. Professora Associada da Faculdade de Medicina de Botucatu. SP/Brasil. https://orcid.org/0000-0002-0090-8524

Recebido em: 10/06/2020

Aprovado em: 11/06/2020

\section{INTRODUÇÃO}

$\Lambda$ segurança do paciente é um componente essencial da qualidade do cuidado dentro de instituições públicas e privadas, e a enfermagem tem papel fundamental no sentido de tornar as atividades laborais seguras e menos arriscadas. Esta preocupação tornou-se assunto de relevância crescente em todo o mundo nos últimos anos, tanto que, em 2004, a Organização Mundial de Saúde (OMS) lançou a
Aliança Global para a Segurança do Paciente (World Alliance for Patient Safety), iniciativa global em prol da melhoria da segurança do paciente em virtude do número alarmante de morbidade e mortalidade em todos os sistemas de saúde, em circunstâncias passíveis de medidas de prevenção $^{(1,2)}$.

A OMS enfatiza que a segurança do paciente é uma questão que afeta os países em todos os níveis de desenvolvimento, sendo que as estimativas deste problema ainda são escassas, principalmente 
em países em desenvolvimento, onde, possivelmente, milhões de pacientes, a cada ano, sofrem lesões incapacitantes ou morrem devido aos erros nos cuidados de saúde ${ }^{(3)}$.

Destaca-se que é importante que os gestores, profissionais de saúde e pesquisadores conheçam as características dos eventos adversos (EAs) evitáveis e que estes podem ser mitigados pela adoção de intervenções que minimizem o risco. E, ainda, que métodos simples podem ser elaborados e testados, buscando monitorar e evitar, em tempo real, os incidentes, os quais têm possibilidade de resultar em prejuízos físicos, emocionais e financeiros para o paciente e para os profissionais envolvidos em seu cuidado ${ }^{(4)}$.

Entretanto, apesar do cuidado em saúde trazer enormes benefícios a todos os envolvidos, a ocorrência de erros é possível, e os pacientes podem sofrer graves consequências, até mesmo o óbito. Contudo, quando implementada a prevenção de forma eficaz, apresenta-se como uma estratégia capaz de diminuir o risco de dano desnecessário ao paciente e aumentar a sua segurança ${ }^{(5)}$.

Nos últimos anos, houve um elevado crescimento de erros dos profissionais de enfermagem no Brasil onde os mesmos estão sendo julgados e punidos com a suspensão do direito de atuar na área. Segundo o Conselho Federal de Enfermagem (COFEN), as principais causas de tais erros, são indicadas por especialistas como sendo a falta de formação adequada e de consciência sobre a função exercida ${ }^{(6)}$.

Muitos são os fatores capazes de causar incidentes ético-profissionais, sejam relacionados à formação profissional, às condições de trabalho, à sobrecarga operacional ou à ausência de situações essenciais ao bom exercício profissional. Entretanto, nenhum desses fatores, ou todos associados, podem justificar o erro profissional, o dano às pessoas ${ }^{(7)}$.

Frente a esse contexto, é imprescindível que novas abordagens do processo de educação devam ser adotadas para garantir o acesso à formação daqueles que ainda não a possuem, como também a educação permanente daqueles que atuam em unidades formadoras de capital humano e prestadoras de serviços de saúde que pretendem ampliar a sua formação profissional.

Sendo assim, nos últimos anos, o ensino profissionalizante brasileiro tem sofrido mudanças significativas, particularmente os cursos de nível técnico que passaram de uma formação essencialmente tecnicista, reprodutivista, direcionada, sobretudo, para a execução de técnicas e o alcance de alguns resultados, para um ensino mais globalizado, com a junção dos saberes, que objetiva atingir determinadas competências para uma atuação mais qualificada, com conhecimentos, habilidades e atitudes para enfrentar os desafios do mercado de trabalho e a segurança do paciente. Essa mudança no modelo do ensino pretendido para o nível técnico se fez presente nas mais diversas áreas profissionais, inclusive na enfermagem ${ }^{(8)}$.

Fazendo oposição ao inatismo e ao empirismo, surge no século 20 o construtivismo (caminho do meio), entendendo que o conhecimento nem seria inerente ao sujeito (apriorismo), muito menos do meio (externo), mas sim de uma interação, o conhecimento iniciaria a partir da interação sujeito-objeto, bem como interação social. As teorias construtivistas surgiram de um esforço na intenção de buscar a complexidade do processo de conhecimento, para tanto, apoiaram-se em pesquisas científicas de diversas áreas - biologia, psicanálise, medicina - para buscar a compreensão do processo cognitivo( ${ }^{(9,10)}$.

As profundas modificações deste mundo contemporâneo, o acúmulo exponencial de conhecimento e a incorporação de novas tecnologias fazem com que o antigo ensino tradicional empirista, nas universidades brasileiras, baseado no professor, com o objetivo de transferir o seu conhecimento para os alunos e avaliar o nível de conhecimento adquirido, seja questionado. Por vezes, na área da saúde, determina-se um sistema educacional tra- dicional com enfoque na ação curativa, individualizada e uni causal da doença, produzindo um ensino dissociado das reais necessidades de saúde vigentes ${ }^{(11-14)}$.

$\mathrm{Na}$ intenção do cumprimento das novas Diretrizes Curriculares Nacionais, e as exigências de um mundo atual interdependente, que demandam uma educação profissional transformadora, os estabelecimentos de ensino necessitam de atualização nos métodos empregados no processo de formação dos estudantes, e, inclusive, com o intuito de atender a esta necessidade, algumas instituições formadoras na área de saúde já vêm propondo mudanças em seus currículos e métodos de ensino-aprendizagem ${ }^{(15,16)}$.

Para tanto, a educação deve ter como preceito a necessidade de acompanhar e intervir criticamente, tendo como uma de suas principais características a utilização das tecnologias digitais e educacionais como forma de aprendizagem. Neste contexto, todas as modalidades de ensino têm passado por transformações, com as tecnologias digitais e educacionais, a aplicação de conceitos, teorias e metodologias mais interativas e dinâmicas estão sendo utilizadas na educação, mudando o foco do processo de ensinar/aprender, seja em cursos e disciplinas totalmente virtuais seja em cursos e disciplinas semipresenciais, realizando a auto aprendizagem.

Os técnicos e os auxiliares representam a maior parte da força de trabalho em Enfermagem e sua formação é de grande importância, pois passam apenas um ou dois anos no ensino profissionalizante e já se inserem como profissionais no mercado de trabalho, assumindo a responsabilidade de cuidar do ser humano em conjunto com outros profissionais de saúde ${ }^{(17)}$.

Assim, diante da necessidade de implantação de processo educativo sobre segurança do paciente nos currículos atuais das instituições de ensino em saúde, levantamos a hipótese de que a organização de um curso extracurricular semipresencial com inserções em cenários de prática curriculares poderia contribuir com a formação dos alunos do curso 
técnico de enfermagem do Centro Paula Souza, além de propiciar um encaminhamento aos gestores de ensino da instituição para inclusão formal deste tema no currículo do curso. Essa proposta pedagógica possibilita uma interação de todos os envolvidos, ampliando a produção do conhecimento em rede.

Diante dessas considerações, almeja-se responder, com o desenvolvimento da pesquisa, a seguinte questão norteadora: Como utilizar e integrar na base curricular obrigatória a abordagem do tema na realização de curso complementar em método Educação a Distância utilizando Plataforma Informatizada Moodle e aulas presenciais com avaliação prática final, como forma de contribuição e qualificação do profissional que será inserido no mercado após a conclusão do curso técnico?

Entende-se que a pesquisa possa contribuir para o campo da enfermagem, uma vez que coloca a atuação destes profissionais como imprescindível para garantir qualidade e segurança aos pacientes. Espera-se também contribuir para o envolvimento de pacientes e seus familiares no processo do cuidado através de cartilha educativa e que este estudo possibilite o desenvolvimento de pesquisas sobre a temática para o reconhecimento de sua importância, tanto no âmbito das organizações hospitalares quanto para profissionais de saúde, em especial a enfermagem.

Desta forma, objetivou-se elaborar e aplicar curso extracurricular semipresencial sobre segurança do paciente aos alunos de curso técnico em enfermagem e criar cartilha educativa para pacientes e familiares.

\section{METODOLOGIA}

Estudo prospectivo caracterizado como investigação exploratória e descritiva, com a finalidade de analisar a retenção do conhecimento de alunos de um curso técnico em enfermagem com a utilização de plataforma informatizada em um curso paralelo a grade curricular.
O curso técnico em enfermagem é composto por quatro módulos, sendo que o primeiro e segundo módulos contemplam os requisitos para certificação de auxiliares de enfermagem e o terceiro e quarto os requisitos para certificação do técnico em enfermagem.
Com enfoque na educação realizada pelo sistema público de nível técnico, o presente estudo foi contextualizado no Centro Paula Souza, autarquia do Governo do estado de São Paulo vinculada à Secretaria de Desenvolvimento Econômico, Ciência e Tecnologia, que apresenta a missão de promover a educação profissional pública dentro de referenciais de excelência, visando ao atendimento das demandas sociais e do mundo do trabalho.

O Centro Paula Souza administra 221 Escolas Técnicas Estaduais (ETEC), distribuídas em 163 municípios paulistas que atendem cerca de 230 mil estudantes nos Ensinos Técnicos, Técnico integrado ao Ensino Médio, distribuídos nos 143 cursos técnicos para os setores Industrial, Agropecuário e de Serviços. Dessas, 43 escolas são de curso técnico em enfermagem. Este número inclui 5 cursos técnicos oferecidos na modalidade semipresencial, 4 cursos técnicos na modalidade on-line, 3 cursos técnicos na modalidade aberta, 29 cursos técnicos integrados ao Ensino Médio e 6 cursos técnicos integrados ao Ensino Médio na modalidade de Educação de Jovens e Adultos (EJA). O projeto político pedagógico (PPP) para esses cursos mostra-se coerente com os Referenciais Curriculares Nacionais da Educação Profissional de Nível Técnico.

O curso técnico em enfermagem é composto por quatro módulos, sendo que o primeiro e segundo módulos contemplam os requisitos para certificação de auxiliares de enfermagem e o terceiro e quarto os requisitos para certificação do técnico em enfermagem. Os alunos que participaram do curso extracurricular estavam no terceiro módulo e nas competências já desenvolvidas tiveram uma abordam do tema "segurança do paciente" de modo superficial.

Cabe ressaltar que segundo o Plano Político Pedagógico, o profissional será considerado técnico em enfermagem pelo Centro Paula Souza quando possuir visão crítica e reflexiva, além de demonstrar habilidades cognitivas, psicomotoras e afetivas, fundamentadas nos conhecimentos 
técnicos e científicos, éticos, políticos e educativos que contribuam para o alcance da qualidade do cuidar em enfermagem.

O estudo foi desenvolvido na Escola Técnica: ETEC - Joaquim Ferreira do Amaral- Jaú - Centro Paula Souza, no curso Técnico de Enfermagem para 46 alunos do $3^{\circ}$ e $4^{\circ}$ módulo a partir do segundo semestre de 2017. A carga horária do curso foi de 30 horas, contemplando 15 horas de aulas e palestra presenciais e 15 horas a distância sendo utilizada plataforma informatizada Moodle. A plataforma Moodle é utilizada na instituição e oferece recursos pedagógicos importantes tais como: facilitação do contato professor aluno, fóruns de discussão, chats, troca de arquivos, monitoramentos dos alunos entre outras.

O curso foi criado pela autora da pesquisa sobre o tema abordado, embasado nos seis passos sobre segurança do paciente de acordo com o Ministério da Saúde ${ }^{(18)}$.

Foram utilizados vídeos aulas gravadas por profissionais da saúde envolvidos com o tema e auxílio do NEAD. TIS - Núcleo de Educação à Distância e Tecnologias da Informação em Saúde - UNESP - Botucatu além de fórum, arquivos de material didático, vídeos, chats, questionários para avaliação, palestra do conselho de classe COREN, entre outros recursos.

Para verificação das competências adquiridas, os alunos que não realizaram (G2) e os que realizaram (G1) o curso específico sobre segurança do paciente, foram submetidos a duas avaliações dissertativas sobre o tema antes do início e após o termino do curso. A correção foi acompanhada por gabarito contendo respostas previamente estabelecidas. Os conteúdos abordados nessa avaliação foram as 6 Metas da Segurança do Paciente do Ministério da Saúde com pontuação máxima de 10 pontos.

A fim de promover o desenvolvimento e o aprimoramento de competências profissionais e na tentativa de equilibrar os processos avaliativos empregados no ensino técnico de enfermagem, para a avaliação das habilidades e atitudes, foi utilizado um processo desenvolvido nos Estados Unidos denominado OSCE (Objective Structured Clinical Examination), sendo exame clínico objetivo estruturado por ter como finalidade avaliar de modo estruturado, objetivo e protegido as habilidades necessárias à prática profissional ${ }^{(19)}$.

\section{Figura 1. Delineamento do estudo. Botucatu, SP, Brasil, 2018}

Convite aos alunos para participação no estudo ou somente nas avaliações pré e pós curso, e assinatura do TCLE

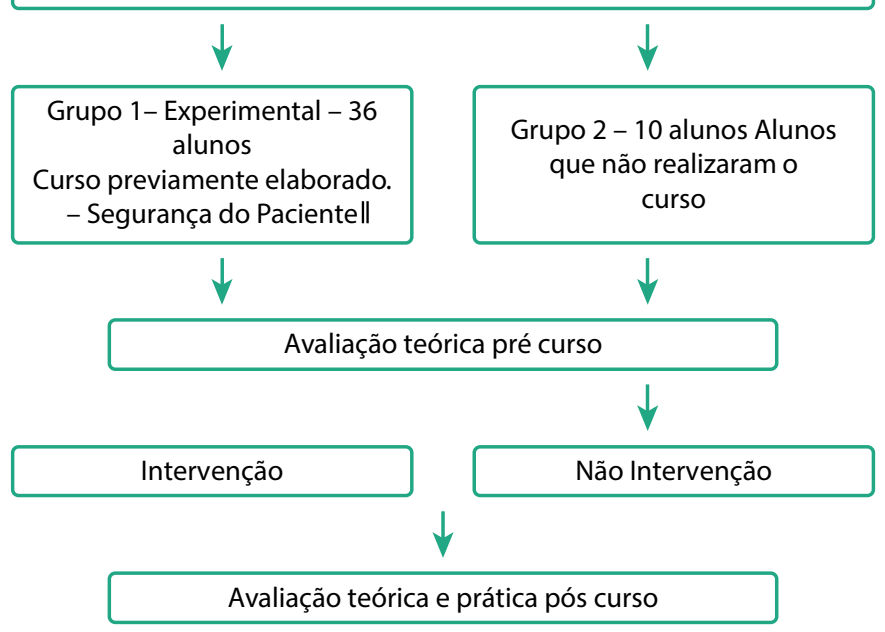

No final do curso, foram realizadas as simulações realísticas tipo OSCE para os 2 grupos (G1 e G2), com quatro estações que continham cenas de situações sobre cirurgia segura, prevenção de queda, higienização das mãos e prevenção de lesão por pressão, que contemplam as metas da Segurança do Paciente, segundo Ministério da Saúde. Examinadores de cada estação verificavam em seu check list, os acertos que os alunos apresentaram no tempo de 10 minutos. O valor da prova prática foi de 60 pontos e continha 41 tópicos, sendo obtida a nota final pela divisão 60 por 41, que resultou no valor de 1,46 por tópico.

Foi utilizado o teste $t$ de student para verificar diferenças entre as medias das notas obtidas nos grupos que realizaram e não realizaram o curso.

Os participantes deste estudo corresponderam ao total de 46 discentes regularmente matriculados no curso Técnico de Enfermagem, sendo 36 alunos do $3^{\circ}$ módulo que realizaram o curso e 10 alunos do $4^{\circ}$ módulo que não participaram do curso, mas auxiliaram para coleta de dados, participando somente das avaliações aplicadas. Utilizou-se o critério em que participariam do curso somente alunos em período avançado do curso técnico em enfermagem em fase pré-conclusão e que já haviam passado por módulos anteriores onde foi abordado superficialmente o tema Segurança do Paciente.

O projeto do estudo foi submetido e aprovado pelo Comitê de Ética em Pesquisa através da Plataforma Brasil sob o parecer n. ${ }^{\circ}$ 1.932.714, seguindo a Resolução n. ${ }^{\circ}$ 466/2012 do Conselho Nacional de Saúde (CNS), que regulamenta os aspectos éticos da pesquisa envolvendo seres humanos. Os alunos foram informados sobre a pesquisa e incluídos no estudo após assinatura do Termo de Consentimento Livre e Esclarecido.

Para auxiliar na construção de uma educação efetiva em segurança do paciente, foi desenvolvida uma cartilha para melhor orientação aos pacientes e seus familiares, onde verificam-se conceitos e 
práticas para uma assistência ao paciente mais segura. Desta forma, espera-se que a mesma forneça elementos capazes de contribuir para a construção do conhecimento e desenvolvimento da assistência prestada à população.

\section{RESULTADOS}

Realizado curso semipresencial extracurricular com a participação de 36 alunos do $3^{\circ}$ módulo do curso técnico em enfermagem com aprovação e interesse dos alunos e com frequência significativa em todas as atividades propostas no curso incluindo tarefas, aulas virtuais, presenciais e avaliações. No período de 12 de agosto a 07 de outubro de 2017.

O curso elaborado na plataforma Moodle é composto por ambientação e nove semanas contendo ambientação com vídeo de boas vindas, material anexo à programação do curso e fórum de notícias.

$\mathrm{Na}$ primeira semana, com a aula inaugural presencial, foram passadas as orientações gerais sobre o curso e abordadas as temáticas de introdução e conceitos que compõem a segurança do paciente: qualidade em saúde e segurança do paciente: aspectos fundamentais; a segurança do paciente como questão estratégica no mundo; antecedentes no Brasil. Todo material da aula foi anexado na plataforma Moodle, na qual tiveram a participação em um fórum de discussão sobre a importância do tema na futura vida profissional dos alunos.

Segunda semana, com a aula presencial, foi apresentada a temática de cultura da segurança do paciente: introdução e conceitos que compõem a segurança do paciente: os desafios do Programa Nacional de Segurança do Paciente; a cultura da segurança como uma questão transversal e multiprofissional; os eixos do Programa Nacional de Segurança do Paciente; investigação de eventos adversos em serviços de saúde; apresentação dos 6 passos da segurança do paciente - Ministério da Saúde. Na plataforma Moodle foram disponibilizados materiais complementares em biblioteca digital sobre a Portaria n. ${ }^{\circ}$ 529, de 01 de abril de 2013, e a RDC n. ${ }^{\circ}$ 36, de 25 de julho de 2013, que contemplam as diretrizes legais da segurança do paciente. Tarefa on-line: pontuação máxima 5 pontos. Pesquisa sobre notícias de problemas relacionados com a segurança do paciente.

$\mathrm{Na}$ terceira semana, aula na plataforma Moodle, $1^{\text {a }}$ Meta do Ministério da Saúde - segurança do paciente: identificar corretamente o paciente. Videoaula - Enfermeiro Alessandro Gabriel Macedo Veiga - Hospital Amaral Carvalho - Jaú. Disponibilização de material complementar para estudos: Protocolo do Ministério da Saúde - identificação do Paciente.Tarefa on-line: pontuação máxima 5 pontos. Em grupos criados em sala, foram realizadas pesquisas na Internet em dois grupos, sendo que o primeiro pesquisou os tipos de identificação do paciente e qualidade e materiais existentes e o outro grupo os custos de materiais para identificação disponíveis no mercado. Após a pesquisa, realizaram uma proposta aos hospitais e instituições de saúde dos produtos com melhor custo/benefício que encontraram.

$\mathrm{Na}$ quarta semana, aula plataforma Moodle, a $2^{\text {a }}$ Meta do Ministério da Saúde - segurança do paciente "Melhorar a comunicação entre profissionais da saúde". Videoaula - Enfermeira Adriane Lopes Hospital Amaral Carvalho - Jaú. Disponibilização de vídeo complementar: propor novas perspectivas é papel da enfermagem na comunicação com o paciente. Tarefa on-line: pontuação máxima 5 pontos. Após os alunos assistirem ao vídeo complementar: propor novas perspectivas é papel da enfermagem na comunicação do paciente, foram realizadas resenhas sobre pontos importantes apresentados.

$\mathrm{Na}$ quinta semana, aula plataforma Moodle, a $3^{\text {a }}$ Meta do Ministério da Saúde - segurança do paciente "Melhorar a segurança na prescrição, no uso e na administração de medicamentos". Videoaula - Farmacêutica Bioquímica - Derli Maria de Souza Lima e Silva - Hospital Amaral Carvalho - Jaú. Disponibilização de material complementar: Protocolo do Ministério da Saúde - medicação segura. Tarefa on-line: pontuação máxima 5 pontos. Após assistirem a videoaula e a leitura do Protocolo do Ministério da Saúde - medicação segura, responderam à questão: "Quais os nove acertos que devemos utilizar para realização de medicação segura?".

$\mathrm{Na}$ sexta semana, aula plataforma Moodle, a $4^{a}$ Meta do Ministério da Saúde - segurança do paciente "Assegurar cirurgia em local de intervenção, procedimentos e pacientes corretos". Videoaula - Enfermeira Gercilene Cristiane Silveira - Hospital Santa Casa de Jaú. Apresentação de modelo de check list de cirurgia segura da OMS.Tarefa on-line: pontuação máxima 5 pontos. Realizada pesquisa na Internet sobre os diferentes modelos de check list de cirurgia segura e cada aluno selecionou um modelo que foi adaptado à realidade do hospital que o elaborou.

$\mathrm{Na}$ sétima semana, aula plataforma Moodle, a $5^{a}$ Meta do Ministério da Saúde - segurança do paciente "Higienizar as mãos para evitar infecções". Vídeo aula - Docente - Giovana Cristina Serra D'Amico - ETEC - Joaquim Ferreira do Amaral - Jaú. Disponibilização de vídeo complementar com técnica de higienização das mãos.Tarefa on-line: pontuação máxima 5 pontos. Atividade em grupo: Grupo 1: criou um vídeo dos 11 passos propostos pelo Ministério da Saúde para higienização das mãos; Grupo 2: criou um vídeo onde os profissionais manuseavam o paciente e, após, não realizando a higienização das mãos, saíam contaminando o ambiente, utensílios e outros pacientes. Neste, utilizaram o guache preto como demonstrativo de contaminação.

$\mathrm{Na}$ oitava semana, aula presencial, Palestra do COREN: Segurança do Paciente - ETEC "Joaquim Ferreira do Amaral". Aula plataforma Moodle, $6^{\text {a }}$ Meta do Ministério da Saúde - segurança do paciente "Reduzir o risco de quedas e lesão por pressão". Videoaula: Prof. a Assistente Dr. ${ }^{a}$ Adriana Polachini do Valle Departamento de Clinica Médica - FMB- Unesp- 
Botucatu. Disponibilização de material complementar de estudo: Protocolo do Ministério da Saúde - prevenção de quedas e vídeo complementar: prevenção de lesão por pressão. Tarefa on-line: pontuação máxima 10 pontos. Realizaram a leitura do Protocolo do Ministério da Saúde sobre prevenção de quedas e listaram os pontos importantes para a prevenção.
Após assistirem ao vídeo complementar sobre lesão por pressão, descreveram as técnicas para a prevenção.

Na nona semana, avaliação Prática: OSCE (Objective Structured Clinical Examination). - pontuação máxima 60 pontos. A avaliação prática com simulações realísticas por meio do OSCE foi realizada com os 2 grupos (G1 e G2) e montadas
Tabela 1. Comparação das médias de avaliações teóricas pré e pós- curso dos grupos de alunos. Test T for equality of means. Botucatu, SP, Brasil, 2018

\section{Group Statistics}

\begin{tabular}{lllll} 
Realização do Curso & & N & Mean & P \\
Pré Curso & G1 & 36 & 4,7083 & \\
\hline Pós Curso & G2 & 10 & 4,5000 & 0,559 \\
\hline & G1 & 36 & 7,2639 & \\
\hline
\end{tabular}

Tabela 2. Comparação das médias de avaliação prática (OSCE) dos grupos de alunos. Test T for equality of means. Botucatu, SP, Brasil, 2018

\begin{tabular}{ccccc}
\multicolumn{2}{c}{ Group Statistics - OSCE } & & & \\
& Realização do Curso & N & Mean & P \\
OSCE & G1 & 21 & 49,9381 & \\
\hline & G2 & 9 & 40,6222 & 0,00
\end{tabular}

\section{Figura 2. Capa da Cartilha Segurança do Paciente. Botucatu, SP, Brasil, 2018}



ISBN [978-85-65318-51-8] quatro estações que continham situações sobre cirurgia segura, prevenção de queda higienização das mãos e prevenção de lesão por pressão. Para cada estação foi elaborado um check list contendo os itens essenciais que deveriam ser respondidos pelos alunos e que seriam observados por examinadores. A duração de cada estação foi de 10 minutos, sendo que um colaborador computava o tempo decorrido em cada estação e avisava com um sinal sonoro o seu término. Em relação à avaliação do curso, os dados foram obtidos com a realização do curso segurança do paciente, a partir das avaliações teóricas realizadas antes e após o curso e da avaliação prática (OSCE).

As comparações entre as notas medias obtidas nas avaliações teóricas e pratica estão demonstradas nas tabelas 1 e 2 .

Verificou-se que houve diferença estatisticamente significante entre os grupos, sendo que o grupo que realizou o curso obteve desempenho melhor quando comparado ao grupo que não realizou o curso e estes dados foram comprovados estatisticamente.

A cartilha com orientações sobre segurança do paciente foi elaborada e editada juntamente com NEAD. TIS - Núcleo de Educação à Distância e Tecnologias da Informação em Saúde - UNESP - Botucatu e serão fornecidas aos hospitais para contribuir com pacientes e familiares na aquisição de conhecimentos sobre segurança. Os conteúdos abordam as seis metas básicas de segurança indicadas pelo Ministério da Saúde e pelo Programa Nacional de Segurança do Paciente ${ }^{(18)}$ e foram organizados com linguagem simples e muitas ilustrações para tornar-se uma leitura acessível e agradável aos pacientes e familiares.

\section{DISCUSSÃo}

O presente estudo teve como objetivo elaborar e implantar curso extracurricular sobre segurança do paciente aos alunos de curso técnico em enfermagem e criar cartilha educativa para pacientes e familiares. 
A abordagem sobre segurança do paciente é um tema recente nos currículos de graduação em saúde. Autores ${ }^{(20)}$ avaliaram quatro cursos da área da Saúde da Universidade Federal de São Paulo (Unifesp), analisando os Projetos Pedagógicos dos cursos de Enfermagem, Farmácia, Fisioterapia e Medicina para identificar os conteúdos sobre segurança do paciente. Verificou-se que, no currículo formal, o ensino sobre segurança do paciente mostrou-se fragmentado, carecendo de aprofundamento e amplitude conceitual.

Diante este contexto, a abordagem especifica sobre segurança do paciente em curso técnico de enfermagem pode contribuir para os necessários avanços nessa área.

O curso foi aplicado de forma semipresencial com 15 horas a distância e 15 horas em salas de aula. As atividades presenciais tiveram como objetivo a introdução ao tema com abordagem nas temáticas: Qualidade em saúde e segurança do paciente: aspectos fundamentais, A segurança do paciente como questão estratégica no mundo, Antecedentes no Brasil, Os desafios do Programa Nacional de Segurança do Paciente, A cultura da segurança como uma questão transversal e multiprofissional, Os eixos do Programa Nacional de Segurança do Paciente, Investigação de eventos adversos em serviço de saúde, Apresentação dos seis passos da segurança do paciente - MS, palestra ministrada pelo conselho de classe COREN e avaliação prática.

As atividades a distância abordaram essencialmente os seis passos sobre segurança do paciente, de acordo com o Ministério da Saúde ${ }^{(18)}$.

O ambiente virtual favoreceu discussões e reflexões sobre o tema, como se pode observar no fórum sobre a importância do tema.

Verificou-se entre as manifestações dos alunos, um reconhecimento da magnitude do problema e um dos alunos diz que:

"Segurança do paciente, é um tema extremamente importante na nossa vida profissional futura, pois é através desse tema que iremos cursar, que passaremos mais segurança ao paciente e iremos nos aprofundar sobre o tema para podermos atuar da melhor maneira possível em prol do paciente e estaremos qualificados para saber o que é o melhor a se fazer em relação ao paciente e quais são os seus direitos dentro do sistema de saúde".

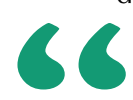

\section{Diante este} contexto, a abordagem especifica sobre segurança do paciente em curso técnico de enfermagem pode contribuir para os necessários
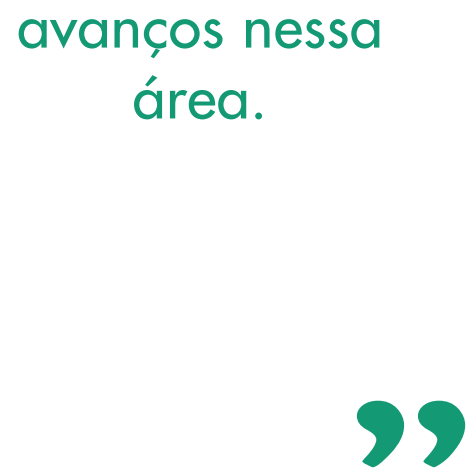

Também se observou, nos relatos dos alunos, uma preocupação com a frequ- ência dos erros e eventos adversos. Em uma de suas citações o aluno relata:

"É importante aprender que os danos/eventos adversos não são só causados por profissionais da saúde, mas por problemas no processo de cuidados em saúde que exigem protocolos e ações que hoje são necessários para cuidar do paciente".

“O curso segurança do paciente é considerado o mais importante quando se fala em qualidade na assistência à saúde, entende-se tudo aquilo que é estudado e aplicado na prática para que os riscos desses danos diminuam até um nível aceitável, ou até mesmo que haja eliminação destes riscos".

“Em minha opinião vai ser de grande valia para minha vida profissional, ter conhecimentos dos fatos e porque eles ocorrem".

Diante estas manifestações, podemos considerar que o objetivo de motivar e sensibilizar os alunos para o tema a ser explorado foi atingido. Com a realização das atividades e tarefas na plataforma Moodle, como leituras dos protocolos vigentes, pesquisas, criação de vídeos, fórum de discussão, videoaulas, entre outros, pudemos observar nos alunos alguns desfechos positivos, como habilidades e conhecimentos, com auxilio das ferramentas facilitadoras da plataforma Moodle.

A plataforma foi bom instrumento, sendo que o Ambiente Virtual de Aprendizagem (AVA) Open Source Moodle (Modular Object Oriented Distance Learning - Objeto Modular Orientado ao Ensino a Distância) é utilizado por instituições de ensino em todo o mundo, por ser um ambiente que não só trata a aprendizagem como uma atividade social, mas focaliza a atenção na aprendizagem que acontece enquanto construímos ativamente artefa- 
tos (como textos, por exemplo), para que outros os vejam ou utilizem ${ }^{(21)}$.

A plataforma Moodle proporcionou interações valiosas nas discussões em grupo com aumento nas interações dos alunos, facilidade de acesso aos conteúdos, as videoaulas eram de curta duração, podendo ser vistas de acordo com a disponibilidade dos alunos e facilidade entre contato professor e aluno e monitoramento para acompanhamento e avaliações.

O curso é viável para outras instituições do Centro Paula Souza para colaboração junto ao PNSP, criando a inclusão do tema de segurança do paciente no ensino e qualificando os alunos do curso técnico em enfermagem para atuação profissional.

A avaliação prática (OSCE) contribuiu para que os alunos conseguissem articular e integrar o processo de atuação segura aos cuidados a partir das cenas criadas como ferramenta de simulação e associação do teórico/prático. A realização da OSCE como meio de avaliação possibilitou a observação do aluno no cenário de simulação e contribuiu para a percepção do aluno de como aplicar os conhecimentos teóricos adquiridos. A experiência dos professores que ajudaram na aplicação da avaliação prática foi muito positiva, acrescentando em seus conhecimentos uma nova maneira de avaliação, próxima da realidade vivenciada na prática em estágios.

O OSCE começou a ser utilizado recentemente nos cursos de enfermagem no Brasil, como forma de aquisição de conhecimento e avaliação, uma vez que os professores posicionam o estudante frente a situações reais de tomadas de decisões e condutas de enfermagem. Isso constitui evidências para a relevância da utilização na enfermagem, o que exige dos envolvidos no processo de ensino-aprendizagem a reflexão em torno do emprego deste método de avaliação.

Estudos $^{(22,23)}$ têm mostrado a efetividade do OSCE na padronização da avaliação das competências dos estudantes nos cursos de Enfermagem presenciais e a distância, utilizando estações específicas para procedimentos técnicos, como exame físico e atividade de raciocínio clínico.

Esses estudos evidenciam que várias universidades estão utilizando o método OSCE como recurso de aprendizagem e como ferramenta de avaliação para identificar pontos fracos em habilidades clínicas e possibilitar um feedback imediato. A integração da avaliação OSCE pode ser compreendida como mecanismo de inovação e, como tal, deve atender às condições de segurança necessárias para que a mudança no curso ocorra. Por esse motivo, deve ser atribuída a devida importância.

Assim, é apontado como elemento fundamental na melhoria do ensino- aprendizagem, podendo ser uma oportunidade de introduzir avaliações de habilidade e atitudes nas ETCs, pois a maioria dos cursos de enfermagem realizam provas práticas, porém não realísticas e estruturais.

No contexto da saúde, o processo educativo consiste muito mais do que o simples ato de ensinar. O cliente, que muitas das vezes erradamente denominamos como indivíduos passivos, é peça chave fundamental no processo de cuidado, uma vez que o processo de cuidar em saúde é dinâmico e requer uma participação de ambas as partes, seja ela cuidadora, ou o indivíduo que receberá o cuidado ${ }^{(24)}$.

A distribuição da cartilha aos pacientes e seus familiares será de relevante importância para a autonomia do paciente e para o gerenciamento dos riscos inerentes no que se refere ao seu cuidado.

Fica evidente que há melhorias no cuidado à saúde quando o paciente recebe instruções sobre segurança. As atividades educativas necessárias para a implantação desse tipo de programa são fatores estratégicos para a mudança de cultura e para a adequação dos $\operatorname{processos}^{(25)}$.

A implantação de um programa de qualidade e acreditação pode trazer significativas contribuições para o serviço, como maior qualidade, maior segurança para o cliente e também para o trabalhador, o qual se sente mais seguro em desempenhar suas atividades. As atividades educativas necessárias para a implanta- 
ção desse tipo de programa são fatores estratégicos para a mudança de cultura e para a adequação dos processos.

A inclusão de curso ou disciplina específica sobre segurança do paciente no currículo formal do curso técnico de enfermagem seria muito relevante já que no currículo vigente da ETEC - Joaquim Ferreira do Amaralll - Jaú este importante assunto é abordado de maneira superficial.

A avaliação deve ser um instrumento no qual se possa identificar e analisar a evolução, o rendimento e as modificações do educando, confirmando a construção do conhecimento. Portanto, foi entendido que a utilização de avaliação pré e do pós curso pode ser uma boa alternativa para rastreio da aprendizagem do participante ao entrar e ao sair do curso.

A enfermagem respalda sua prática por meio de evidências e do conhecimento científico e, para que a qualidade e a segurança na assistência em enfermagem sejam contínuas, é importante o profissional se sentir seguro no que faz. Para isso, são necessários estudo e atualização constantes.

Para a melhoria na assistência de saúde, são necessários empenho e comprometimento da equipe para atingir sempre a satisfação do paciente. Tais ações se complementam com treinamentos e educação para o serviço; dessa forma, o profissional consegue se preparar e conferir mais confiança na assistência prestada, melhorando a qualidade do serviço.

\section{CONCLUSÃO}

A aplicação do curso segurança do paciente em plataforma informatizada Moodle contribuiu com a melhoria da qualidade do ensino técnico de enferma- gem neste tema. Essa proposta pedagógica possibilitou uma interação entre todos os envolvidos, ampliando para a produção do conhecimento em rede.

A cartilha com orientações aos pacientes e seus familiares sobre segurança do paciente favorecerá a implantação da autonomia do paciente e contribuirá para uma assistência mais segura.

Conclui-se, portanto, que a utilização do método Educação a Distância com a Plataforma Informatizada Moodle traz contribuições positivas para o desenvolvimento das competências previstas no plano de curso, sendo que este curso específico de segurança do paciente, já validado, poderá alcançar maior abrangência nas unidades de ensino do Centro Paula Souza do Estado de São Paulo, contribuindo para qualificação profissionais dos alunos que formamos no curso técnico em enfermagem.

\section{Referências}

1. Martinez AA, Montoro CH, González MG. Fortalezas e ameaças em torno da segurança do paciente segundo a opinião dos profissionais de enfermagem. Rev. Lat. Am. Enfermagem. 2010; 18(3):[08 telas].

2. Martinez AA, Vázquez AA, Campo M. El cuidado y La seguridade de Ipaciente: algunas consideraciones éticas y legales. Ética Cuid. 2008; 1(1).

3. World Health Organization. Summary of the evidence on patient safety: implications for research: The Research Priority Setting Working Group of the World Alliance for Patient Safety. Geneva:WHO, 2008.

4. Mendes W, et al. Características de eventos adversos evitáveis em hospitais do Rio de Janeiro. Rev. Assoc. Med. Bras. 2013; 59(5):421-428.

5. Carvalho SHDBC, et al. Clima de segurança do paciente: percepção dos profissionais de enfermagem. Acta Paul. Enferm. 2012; 25(5):728-735.

6. Conselho Regional de Enfermagem de São Paulo (BR). Conselho Federal de Enfermagem. Anotações de Enfermagem. São Paulo: COREN-SP, 2011. 22 p.

7. Carvalho VT, Cassiani SHB. Erros na medicação e consequências para profissionais de enfermagem e clientes: um estudo exploratório. Rev. Lat. Am. Enfermagem. 2002; 10(4):523-529.

8. Ferreira-Júnior MA, Grígoli JAG, Ivo ML. 0 ensino por competências na área da enfermagem: interpretações e práticas pedagógicas. Rev. Enferm. Cent. 0. Min. 2011 abr/jun; 1(2):143-153.

9. Pontes AL, Rego S, Silva-Júnior AG. Saber e prática docente na transformação do ensino médico. Rev. Bras. Educ. Méd. 2006; 30(2):66-75.

10. Aranha ML. A. História da educação e da pedagogia. 3. ed. São Paulo: Moderna; 2010.

11. Souza CS, Iglesias AG, Pazin-Filho A. Estratégias inovadoras para métodos de ensino tradicionais - aspectos gerais. Medicina (Ribeirão Preto). 2014; 47(3):284-92.

12. Harts AM, Schlatter GV. A construção do trabalho de conclusão do curso por meio da metodologia ativa team-basedlearning. Adm. Ensino Pesqui. 2016; 17(1):73.

13. Mitre SM, et al. Metodologias ativas de ensino-aprendizagem na formação profissional em saúde: debates atuais Active teaching-learning methodologies in health education: current debates. Ciênc. Saúde Colet. 2008; 13(2):2133-2144.
14. Sobral FR, Campos CJG. The use of active methodology in nursing care and teaching in national productions: An integrative review. Rev. Esc.Enferm. 2012; 46(1):208-218.

15. Bollela VR, et al. Aprendizagem baseada em equipes: da teoria à prática. Medicina (Ribeirão Preto). 2014; 47(3):293-300.

16. Honda K, Chirelli MQ. Residência multiprofissional em saúde: formação com metodologias ativas de ensino- aprendizagem desenvolvimento curricular e didática. Indag. Didact. 2015; 7(3):50-61.

17. Orosco SS, Scheide TJF. As diferentes abordagens do processo educativo e seus reflexos no ensino de enfermagem em nível médio. Colloquium (Presidente Prudente). 2008; 5(1):53-68.

18. Ministério da Saúde (BR). Portaria MS/GM n. ${ }^{\circ} 529$, de $1^{\circ}$ de abril de 2013. Institui o Programa Nacional de Segurança do Paciente (PNSP). Brasília: MS, 2013.

19. Medeiros SB, et al. Exame clínico objetivo estruturado: reflexões sob um olhar da enfermagem. Cogitare Enferm. 2014; 19(1):170-173.

20. Bohomol E, Freitas MAO, Cunha ICKO. Ensino da segurança do paciente na graduação em saúde: reflexões sobre saberes e fazeres. Interface. 2016 jul/set; 20(58).

21. Santana DA. 0 uso da plataforma Moodle na Educação à Distância como forma de democratizar o ensino. Web artigos. 2009.

22. McWillian PL, Botwinski CA. Identifying Strengths and weaknesses in the utilization of objective. Structured clinical. Examination (OSCE) in a nursing program. Nurs Educ Perspect. 2012; 33(1):35-9.

23. Oranye NO, Ahmad C, Ahmad N, Bakar RA. Assessing nursing clinical skills competence through objective structured clinical examination. (OSCE) for open distance leaming students in Open University Malaysia. Conthnp nurse. 2012; $41(2): 233-41$.

24. Alvim NAT, Ferreira MA. Perspectiva problematizadora da educação popular em saúde e a enfermagem. Texto Contexto Enferm. 2007; 16(2):315-19. 25. Franco JN, Barros BPA, Vaidotas M, D'Innocenzo M. Percepção dos enfermeiros sobre os resultados dos indicadores de qualidade na melhoria da prática assistencial. Rev Bras Enferm. 2010; 63(5):806-10. 